\title{
Expression of B7-H3 Correlates with PD-LI and Poor Prognosis in Patients with Cervical Cancer
}

\author{
Qianqian Zhang ${ }^{1,2}$ \\ Liju Zong ${ }^{3}$ \\ Hui Zhang ${ }^{2}$ \\ Wei Xie ${ }^{4}$ \\ Fan Yang 5 \\ Wenwen Sun ${ }^{5}$ \\ Baoxia Cui \\ Youzhong Zhang' \\ 'Department of Obstetrics and \\ Gynecology, Qilu Hospital of Shandong \\ University, Jinan, People's Republic of \\ China; ${ }^{2}$ Department of Obstetrics and \\ Gynecology, The Second Affiliated \\ Hospital of Shandong First Medical \\ University, Tai'an, People's Republic of \\ China; ${ }^{3}$ Department of Pathology, Peking \\ Union Medical College Hospital, Chinese \\ Academy of Medical Sciences, Beijing, \\ People's Republic of China; ${ }^{4}$ Department \\ of Emergency, The Second Affiliated \\ Hospital of Shandong First Medical \\ University, Tai'an, People's Republic of \\ China; ${ }^{5}$ Department of Pathology, The \\ Second Affiliated Hospital of Shandong \\ First Medical University, Tai'an, People's \\ Republic of China
}

\begin{abstract}
Purpose: The purpose of this study was to investigate B7 homolog 3 (B7-H3) expression patterns and define its associations with programmed cell-death ligand 1 (PD-L1), pathological features, and survival in patients with cervical cancer.

Patients and Methods: Immunohistochemical staining was used to investigate B7-H3 and PD-L1 expression in tissue microarrays from 552 patients with stage IB1 and IIA1 cervical cancer, including 406 with squamous cell carcinoma and 146 with endocervical adenocarcinoma.

Results: B7-H3 was expressed in the tumor cells (TCs) of $32.1 \%$ of the samples as well as in the stromal cells of $92.9 \%$ of the specimens. B7-H3 was co-expressed with PD-L1 in $21.0 \%$ of the samples, while only one or the other was expressed in $41.7 \%$ of the samples. B7-H3 expression in TCs was more frequent in squamous cell carcinoma, PD-L1-positive samples, and tissues from patients with lymph node metastasis; moreover, its expression was an independent predictor of shorter survival.
\end{abstract}

Conclusion: B7-H3 positivity in TCs is a promising prognostic biomarker, and targeting B7-H3 alone or in combination with PD-1/PD-L1 may be a potential immunotherapeutic strategy for patients with cervical cancer.

Keywords: carcinoma of the cervix, programmed cell-death ligand 1, B7 homolog 3, immunotherapy

\section{Introduction}

Cervical cancer is the third most common cancer in women worldwide. Following the implementation of cervical cancer screening and development of effective preventative vaccines, the overall incidence of cervical cancer has declined during the past decade; ${ }^{1}$ however, the relative and absolute incidences of adenocarcinoma are still rising. ${ }^{2}$ Most patients with early-stage disease have favorable prognoses; nonetheless, those with advanced or recurrent disease have poor outcomes.

In recent years, immunotherapy targeting the immune checkpoint programmed cell-death 1 (PD-1) and its ligand programmed cell-death ligand 1 (PD-L1) has emerged as a novel modality in oncology and has been shown to be effective against a variety of tumor types. The United States Food and Drug Administration has approved the PD-1 inhibitor pembrolizumab for patients with recurrent or metastatic cervical cancer whose tumors express PD-L1 with a combined positive score (CPS) of $\geq 1$. However, the objective response rate for pembrolizumab was found to be $14.6 \%$ in a Phase II study. ${ }^{3}$ Therefore, efforts continue to identify new targets and develop novel immunotherapeutic strategies for patients with this disease.
Correspondence: Youzhong Zhang Department of Obstetrics and Gynecology, Qilu Hospital, Shandong University, Wenhua Xi Road 107\#, Jinan, 2500 I2, People's Republic of China $\mathrm{Tel} / \mathrm{Fax}+86$ 53I-882I 69268

Email zhangyouzhong@sdu.edu.cn 
B7 homolog 3 (B7-H3), also known as CD276, belongs to the B7 superfamily that also includes PD-L1 and is an immune checkpoint protein that exhibits essential roles in T cells. Notably, B7-H3 has been reported to have both costimulatory and coinhibitory abilities in different tumor microenvironments. ${ }^{4}$ Chapoval et al demonstrated that B7$\mathrm{H} 3$ increases the proliferation of $\mathrm{CD} 4+$ and $\mathrm{CD} 8+\mathrm{T}$ cell populations and upregulates IFN- $\gamma$ production. ${ }^{5}$ In contrast, Suh et al showed that $\mathrm{B} 7-\mathrm{H} 3$ is a negative regulator that inhibits $\mathrm{T}$ cell proliferation. ${ }^{6} \mathrm{~B} 7-\mathrm{H} 3$ protein is present at low levels in the liver, colon, and prostate but is rare in normal tissues overall; nevertheless, it is expressed in various types of malignant tissues including lung cancer, ${ }^{7}$ colorectal cancer, ${ }^{8}$ prostatic cancer, ${ }^{9}$ renal cell carcinoma, ${ }^{10}$ breast cancer, ${ }^{11}$ endometrial cancer, ${ }^{12}$ ovarian cancer, ${ }^{13}$ gestational trophoblastic neoplasia, ${ }^{14}$ and cervical cancer. ${ }^{15-18}$ B7-H3 is expressed in tumor cells (TCs) and stromal cells including tumor-associated vascular endothelial cells, fibroblasts, and immune cells. ${ }^{7,19,20}$ Generally, B7-H3 is associated with tumor aggressiveness, unfavorable clinicopathological features, and poor outcomes. It has been shown to promote invasion, migration, and angiogenesis in lung, colorectal, ovarian, and prostate cancers. ${ }^{21-24}$ These results suggest that B7-H3 may be able to induce cancer progression and metastasis beyond its ability to modulate tumor immunity. ${ }^{4}$

Although B7-H3 expression in cervical cancer has been reported in previous studies, including ours, ${ }^{12-15,18}$ its association with PD-L1 remains unclear. Furthermore, the numbers of patients included in previous studies were small, particularly those with adenocarcinoma. Therefore, the associations between B7-H3 and histological subtypes as well as with survival outcomes remain unknown. As such, the aims of this study were to explore B7-H3 expression within cervical cancer tissues and to assess the relationship between this expression and PD-L1, clinicopathological features, and patient outcomes.

\section{Materials and Methods Study Cohort and Tissue Microarray (TMA) Construction}

This retrospective study included 552 radical hysterectomy specimens from patients diagnosed with either squamous cell carcinoma of the cervix $(\mathrm{N}=406)$ between January 2012 and December 2015 or usual type endocervical adenocarcinoma $(\mathrm{N}=146)$ between January 2008 and December 2017, at Qilu Hospital of Shandong
University (Jinan, China). All patients in this study were diagnosed before 2018; therefore, we used the 2009 International Federation of Gynecology and Obstetrics (FIGO) staging system for carcinoma of the cervix. Considering the excellent odds of survival in patients with stage IA, we included only patients with IB1 and IIA1 disease, for whom the standard treatment was radical hysterectomy with pelvic lymph node dissection and/or para-aortic lymph node sampling or dissection. The following patients were excluded from the study: those with non-usual types of endocervical adenocarcinoma (adenosquamous, mucinous gastric/intestinal, adenoma malignum, serous, clear cell, or endometrioid), those with neuroendocrine carcinoma, those with insufficient data, those who died within 1 month after surgery, and those who received neoadjuvant therapy before surgery. Clinicopathologic data (collected from medical records) included age at diagnosis, tumor size, tumor histology, differentiation grade, depth of invasion, parametrial involvement, lymphovascular space invasion, number of positive lymph nodes, postoperative adjuvant treatment (chemotherapy, chemoradiotherapy, or radiotherapy), date of recurrence or last follow-up, and patient status on the last follow-up date. Representative areas within the tumor tissue were marked on hematoxylin and eosin-stained slides and sampled for the TMA blocks. TMAs with a single $2 \mathrm{~mm}$ core per case were constructed using a tissue arrayer (Mini Core, Mitogen, Hertford, UK). This study conformed to the ethical standards set forth in the Declaration of Helsinki and in national and international guidelines and was approved by the Institutional Review Board of Qilu Hospital of Shandong University (approval No. KYLL2017-560). All investigators signed patient data confidentiality statements, and patient data were only accessible on confidential computers at Qilu Hospital of Shandong University. All patients, upon admission to the hospital, signed consent forms allowing review of their medical records, the use of their tissue samples, and possible publication of associated reports. None of the patients can be identified from their clinical information or images in this retrospective study, and informed consent to determine PD-L1 and B7-H3 expression was not required by the Institutional Review Board of Qilu Hospital of Shandong University.

\section{Immunohistochemistry and Assessment}

Immunohistochemistry was performed using our laboratory protocol as described previously. ${ }^{14,17,26}$ Briefly, 4 
$\mu \mathrm{m}$ TMA serial sections were deparaffinized and subjected to heat-induced epitope retrieval with $10 \mathrm{mM}$ sodium citrate $(\mathrm{pH} 6.0)$ at $95^{\circ} \mathrm{C}$ for $20 \mathrm{~min}$. The endogenous peroxidase activity was quenched using a $0.3 \%$ hydrogen peroxide solution. TMA sections were incubated with primary antibodies against PD-L1 (dilution 1:200, clone E1L3N, Cell Signaling Technology, Danvers, USA) and B7-H3 (dilution 1:200, clone D9M2L, Cell Signaling Technology, Danvers, USA). Human placental tissues treated with primary antibodies were used as positive controls, while the same tissues without primary antibodies comprised negative controls. All the slides were stained using an automatic immunohistochemistry staining instrument (BOND-III; Leica Biosystems, Wetzlar, Germany) according to the manufacturer's instructions. PD-L1 was evaluated using the CPS, which was calculated as the sum of the number of PD-L1-stained cells (TCs, lymphocytes, and macrophages) divided by the total number of viable tumor cells, with the quotient multiplied by 100 . A CPS of $\geq 1$ was considered indicative of positive PD-L1 expression. Samples were considered positive for B7-H3 when $\geq 5 \%$ of the TCs expressed this protein at any intensity; the $5 \%$ cut-off was chosen based on a previous publication. ${ }^{6}$ Staining of $\mathrm{B} 7-\mathrm{H} 3$ in stromal fibroblasts, endothelial cells, and immune cells was also recorded.

\section{Statistical Analysis}

The $\chi^{2}$ test was used to determine the association between categorical variables. Relapse-free survival (RFS) was defined as the interval between the date of surgery and that of the detection of the first local, regional, and/or distant relapse. Overall survival (OS) was defined as the time between the date of surgery and that of death from any cause, censoring, or the last follow-up. Survival curves were plotted using the Kaplan-Meier method and compared using the Log rank test. To identify prognostic predictors, univariate and multivariate survival analyses were performed using the Cox proportional hazards regression model, and hazard ratios (HRs) with 95\% confidence intervals (CIs) for recurrence and death were calculated. All statistical analyses were conducted using the Statistical Package for the Social Sciences software (version 20.0; IBM Corp., Armonk, NY, USA). A 2-sided $P$-value $<0.05$ was considered statistically significant.

\section{Results}

\section{B7-H3 Expression in Cervical Cancer}

The median age of the 552 patients in this study was 47 years (range, 24-78 years); their clinicopathological characteristics are summarized in Table 1. A total of 460 patients received postoperative adjuvant treatment, of whom 220 received chemoradiotherapy, 146 received radiotherapy only, and 94 received adjuvant chemotherapy.

B7-H3 was expressed by both TCs and stromal cells and exhibited a cytoplasmic/membranous staining pattern (Figure 1). B7-H3 expression was positive in the TCs of 177 samples (32.1\%) and in the stromal cells of 513 (92.9\%). PD-L1 expression was positive in $51.6 \%$ of the samples (285/552), and was detected in TCs, lymphocytes, and macrophages (Figure 1). Representative immunohistochemical staining of B7-H3 in macrophages, endothelial cells, and stromal fibroblasts is shown in Figure 2.

\section{Association Between B7-H3 Expression in TCs and Clinicopathological Parameters}

The associations between B7-H3 expression in TCs, PD$\mathrm{L} 1$, and clinicopathologic characteristics are presented in Table 1. B7-H3 positivity in TCs was significantly more frequent in squamous cell carcinoma than in adenocarcinoma $(36.0 \%$ vs $21.2 \%)$, and in patients with lymph nodal metastasis than in those without $(40.5 \%$ vs $29.9 \%)$. One hundred and sixteen samples $(21.0 \%)$ exhibited coexpression of B7-H3 and PD-L1, 206 (37.3\%) were double negative, and 230 (41.7\%) were positive for either PD-L1 or B7-H3 alone. Additionally, B7-H3 positivity in TCs was observed more frequently in PD-L1-positive samples than in PD-L1-negative counterparts $(40.7 \%$ vs $22.8 \%)$. No statistically significant differences were observed between B7-H3 in stromal cells and PD-L1 expression, nor were there significant associations between B7-H3 expression in stromal cells and clinicopathological parameters (data not shown). In addition, PD-L1 positivity was more frequent in squamous cell carcinoma than in adenocarcinoma $(58.4 \%$ vs $32.9 \%, P<0.001)$ and in poorlydifferentiated tumors compared to well-to-moderately differentiated tumors $(55.7 \%$ vs $43.5 \%, P=0.007)$. Patients with PD-L1-positive tumors and those with positive B7$\mathrm{H} 3$ expression in TCs were more likely to have received postoperative adjuvant treatment than patients with $\mathrm{PD}-\mathrm{L} 1$ -negative $(89.8 \%$ vs $76.4 \%, P<0.001)$ or $\mathrm{B} 7$-H3-negative tumors $(88.1 \%$ vs $81.1 \%, P=0.038)$. 
Table I Clinicopathological Characteristics and Expression Patterns of B7-H3 in Patients with Cervical Cancer ( $\mathrm{N}=552)$

\begin{tabular}{|c|c|c|c|c|}
\hline \multirow[t]{2}{*}{ Variables } & \multirow[t]{2}{*}{$\mathbf{N}(\%)$} & \multicolumn{2}{|c|}{ B7-H3 } & \multirow[t]{2}{*}{$P$-value } \\
\hline & & Negative (N) & Positive (N, \%) & \\
\hline Age & & & & 0.552 \\
\hline$<45$ years & $227(4 I . I)$ & $|5|$ & $76(33.5)$ & \\
\hline$\geq 45$ years & $325(58.9)$ & 224 & I0I (3I.I) & \\
\hline FIGO stage & & & & 0.717 \\
\hline$|\mathrm{B}|$ & $463(83.9)$ & 316 & $147(3 \mid .7)$ & \\
\hline$\| \mathrm{IAI}$ & $89(16.1)$ & 59 & $30(33.7)$ & \\
\hline Histology & & & & 0.001 \\
\hline Squamous cell carcinoma & $406(73.6)$ & 260 & $146(36.0)$ & \\
\hline Adenocarcinoma & $146(26.4)$ & 115 & $3 \mid(2 \mid .2)$ & \\
\hline Differentiation & & & & 0.945 \\
\hline Well to moderate & $186(33.7)$ & 126 & $60(32.3)$ & \\
\hline Poor & $366(66.3)$ & 249 & $117(32.0)$ & \\
\hline Depth of invasion & & & & 0.894 \\
\hline$<1 / 2$ & I58 (28.6) & 108 & $50(31.6)$ & \\
\hline$\geq \mathrm{I} / 2$ & $394(71.4)$ & 267 & $127(32.2)$ & \\
\hline LVSI & & & & 0.436 \\
\hline No & $432(78.3)$ & 297 & I35 (3I.3) & \\
\hline Yes & $120(21.7)$ & 78 & $42(35.0)$ & \\
\hline Nodal metastasis & & & & 0.032 \\
\hline Negative & $44 \mid(79.9)$ & 309 & 132 (29.9) & \\
\hline Positive & III (20.I) & 66 & $45(40.5)$ & \\
\hline Parametrial involvement & & & & 0.482 \\
\hline Yes & $539(97.6)$ & 365 & $174(32.3)$ & \\
\hline No & $13(2.4)$ & 10 & $3(23.1)$ & \\
\hline PD-LI & & & & $<0.001$ \\
\hline Negative & $267(48.4)$ & 206 & $61(22.8)$ & \\
\hline Positive & $285(5 \mathrm{I} .6)$ & 169 & $116(40.7)$ & \\
\hline
\end{tabular}

Abbreviations: B7-H3, B7 homolog 3; FIGO, International Federation of Gynecology and Obstetrics; LVSI, lymphovascular space invasion; PD-LI, programmed cell-death ligand I.

\section{Associations of B7-H3 Expression in TCs with Patient Outcomes}

After a median follow-up of 55 months (range, 7-114 months), 54 patients $(9.8 \%)$ relapsed and $61(11.1 \%)$ died of any cause. Kaplan-Meier survival analyses showed that patients who had B7-H3-positive TCs had a shorter RFS and OS (Figure 3). In univariate analysis, B7-H3 positivity in TCs, FIGO stage IIA1, parametrial involvement, deep invasion ( $\geq 1 / 2)$, and lymph node metastasis were associated with a shorter RFS and OS (Table 2). In multivariate analysis, $\mathrm{B} 7-\mathrm{H} 3$ positivity in TCs was a predictor of shorter RFS (HR 2.1, 95\% CI 1.2-3.6, $P=$ 0.006 ) and OS (HR 1.8, 95\% CI 1.1-3.0, $P=0.023$ ), independent of FIGO stage or lymph nodal metastasis (Table 3). Positive PD-L1 or B7-H3 expression in stromal cells was not associated with patient outcomes.

\section{Discussion}

To the best of our knowledge, ours is the first study to investigate B7-H3 expression and its association with PDL1, clinicopathological features, and survival in a large cohort of patients with cervical cancer. We observed tumoral $\mathrm{B} 7-\mathrm{H} 3$ expression in $32.1 \%$ of the patients; this protein was also expressed in the stromal cells of most patients. Moreover, B7-H3, PD-L1, or both together were expressed in $62.7 \%$ of the patients. Additionally, B7-H3 
SCC
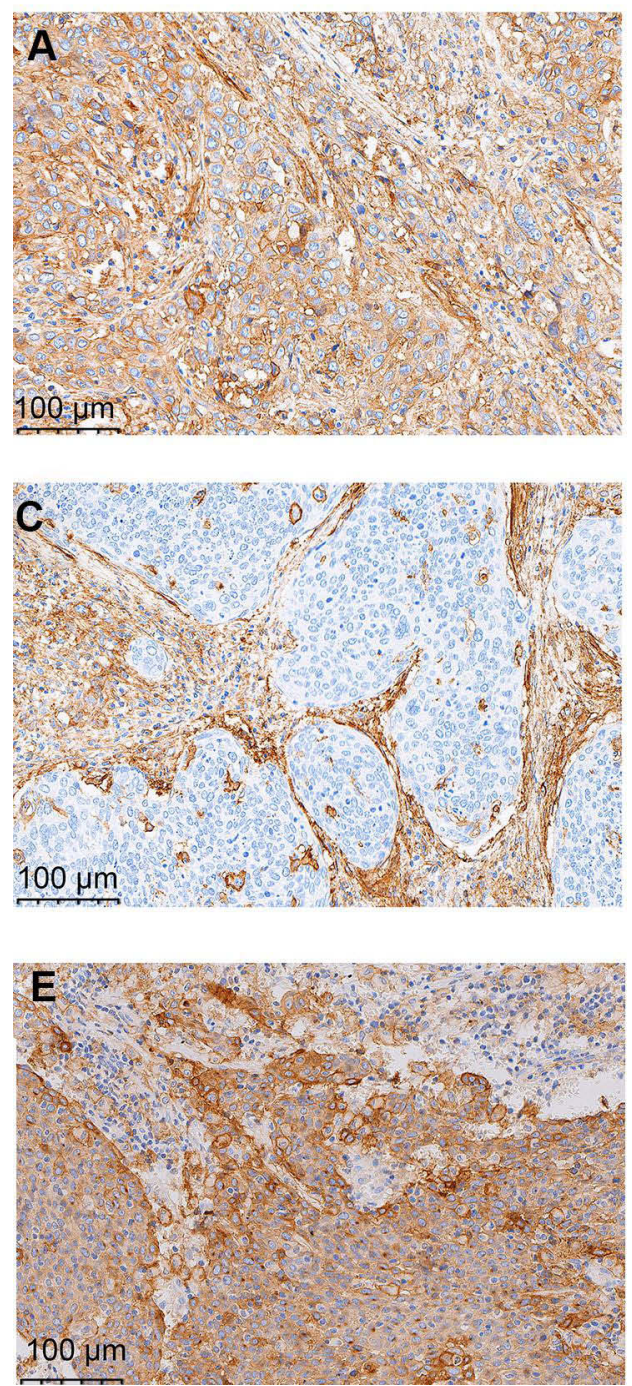

$\mathrm{AC}$
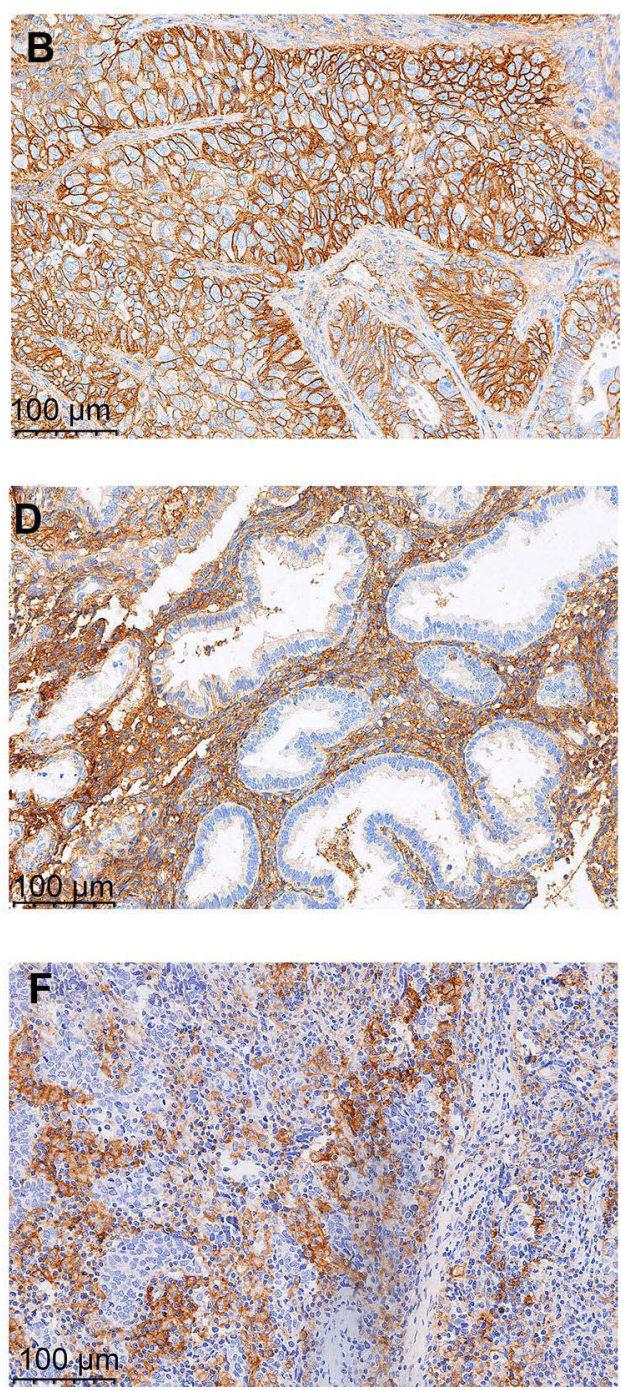

Figure I Representative immunohistochemical staining of B7 homolog 3 (B7-H3) and programmed cell-death ligand I (PD-LI) in squamous cell carcinoma (SCC) (left column) and adenocarcinoma (AC) (right column) of the cervix. Positive B7-H3 in tumor cells and stromal cells (A and B), negative B7-H3 in tumor cells and positive B7-H3 in stromal cells ( $\mathbf{C}$ and $\mathbf{D}$ ), and positive PD-LI as defined by a combined positive score of $\geq I$ ( $\mathbf{E}$ and $\mathbf{F}$ ).

positivity was significantly associated with the histologic subtype, lymph node metastasis, and PD-L1 positivity, and was an independent predictor of shorter RFS and OS. These findings support the notion that $\mathrm{B} 7-\mathrm{H} 3$ is a prognostic marker as well as a therapeutic target (either via monotherapy or combined with PD-1/PD-L1 blockade) in patients with cervical cancer.

Studies investigating B7-H3 expression in cervical cancer are relatively rare. Brustmann et al found that B7-H3 was expressed in most of the 64 samples obtained from patients with cervical squamous cell carcinoma. The protein was expressed in the endothelial cells of the tumorassociated vasculature as well as in epithelial TCs, where its high expression was observed more frequently in patients with FIGO stage IB disease than in those with stage IA; however, its expression was not associated with survival. ${ }^{25}$ In a study of 108 patients, Huang et al demonstrated that B7-H3 was expressed in the TCs of $72 \%$ of their cervical cancer samples; the protein was highly expressed in the stromal compartments, and patients with a high level of B7-H3 expression in TCs had worse prognoses. ${ }^{15}$ In our previous study of a small cohort of 60 patients, we found that $\mathrm{B} 7-\mathrm{H} 3$ positivity correlated with shorter disease-free survival. ${ }^{16}$ Consistent with previously published data, our present study showed that B7-H3 expression was observed in both TCs and stromal cells in patients with cervical cancer, and that its expression in TCs correlated with shorter survival. 
A

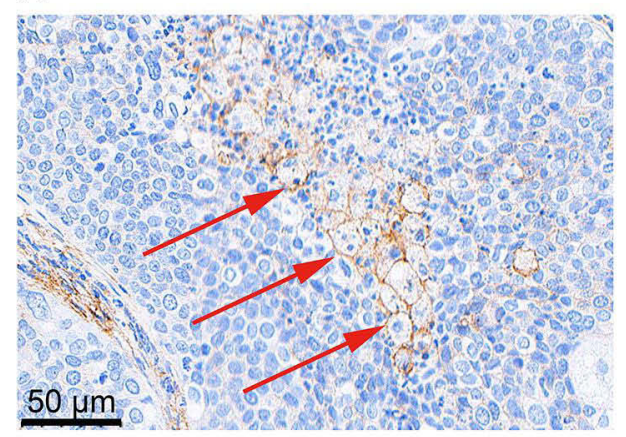

C

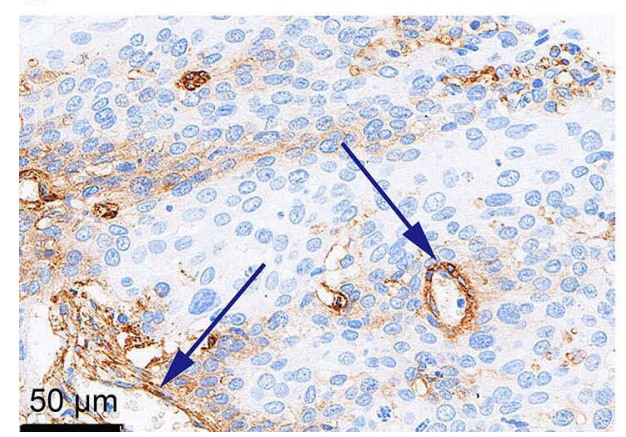

B

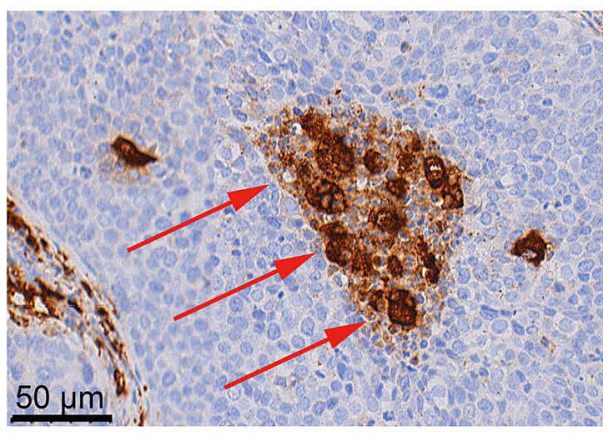

D

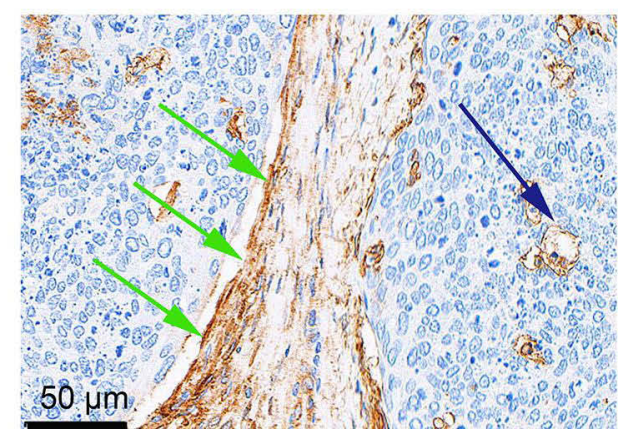

Figure 2 Representative immunohistochemical staining of B7 homolog 3 (B7-H3) in macrophages, endothelial cells, and stromal fibroblasts. B7-H3 expression in (A) macrophages (red arrowheads) and (B) corresponding CD68+ macrophages (red arrowheads); (C) endothelial cells (blue arrowheads); (D) stromal fibroblasts (green arrowheads) and endothelial cells (blue arrowhead).

A

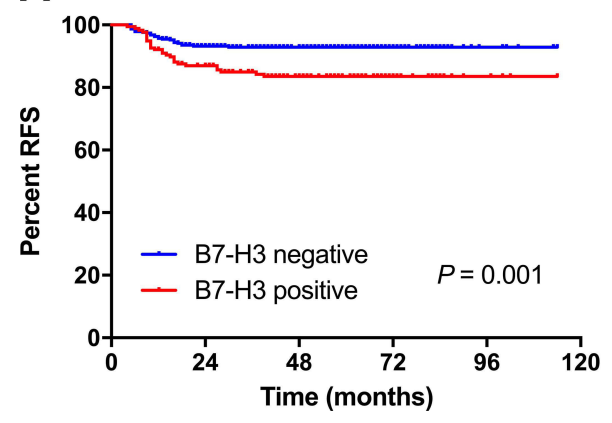

B

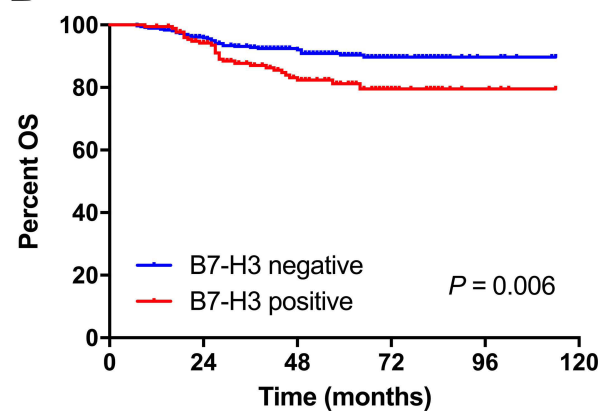

Figure 3 Kaplan-Meier curves showing the (A) relapse-free survival (RFS) and (B) overall survival (OS) of patients with cervical cancer according to B7 homolog 3 (B7-H3) expression in tumor cells.

B7-H3 was expressed more frequently in squamous cell carcinoma than in adenocarcinoma in our cohort. Similarly, Heeren et al demonstrated that squamous cell carcinomas were more frequently positive for PD-L1 than were adenocarcinomas. ${ }^{27}$ No association between B7-H3 expression and histologic subtypes was found in previous studies; this discrepancy may be attributed to their small number of patients with adenocarcinoma. Our study is the first to describe the differential distributions of B7-H3 across histologic subtypes; however, further studies are necessary to confirm our results.
While the expression of PD-L1 and B7-H3 in cervical cancer has previously been reported, ${ }^{15,16,25,27-29}$ none of these studies investigated the potential correlations between PD-L1 and B7-H3 expression. In our study, we observed these proteins' co-expression in $21.0 \%$ of samples, with another $41.7 \%$ containing either one or the other. Moreover, we found that B7-H3 positivity was correlated with PD-L1 expression. Similar results have been reported in lung cancer and upper tract urothelial carcinoma. ${ }^{30-32}$ For example, Altan et al observed that B7-H3 and PD-L1 were co-expressed in $17.6 \%$ of 
Table 2 Univariate Analysis of Relapse-Free and Overall Survival Among Patients with Cervical Cancer $(\mathrm{N}=552)$

\begin{tabular}{|c|c|c|c|c|c|c|}
\hline \multirow[t]{2}{*}{ Variables } & \multicolumn{3}{|c|}{ Relapse-Free Survival } & \multicolumn{3}{|c|}{ Overall Survival } \\
\hline & HR & $95 \% \mathrm{Cl}$ & $P$-value & HR & $95 \% \mathrm{Cl}$ & $P$-value \\
\hline \multicolumn{7}{|l|}{ FIGO stage } \\
\hline IIAI vs IBI & 3.9 & $2.3-6.8$ & $<0.001$ & 3.4 & $2.0-5.7$ & $<0.001$ \\
\hline \multicolumn{7}{|c|}{ Parametrial involvement } \\
\hline Yes vs no & 4.9 & $1.9-12.2$ & 0.001 & 5.3 & $2.3-12.3$ & $<0.001$ \\
\hline \multicolumn{7}{|l|}{ Depth of invasion } \\
\hline$\geq 1 / 2$ vs $<1 / 2$ & 3.4 & $1.4-7.9$ & 0.005 & 3.2 & I.5-7.1 & 0.004 \\
\hline \multicolumn{7}{|l|}{ Nodal metastasis } \\
\hline Positive vs negative & 5.2 & $3.0-8.8$ & $<0.001$ & 4.4 & $2.7-7.5$ & $<0.001$ \\
\hline \multicolumn{7}{|l|}{$\mathrm{B} 7-\mathrm{H} 3$} \\
\hline Positive vs negative & 2.4 & $1.4-4.0$ & 0.02 & 2.0 & $1.2-3.3$ & 0.007 \\
\hline
\end{tabular}

Abbreviations: B7-H3, B7 homolog 3; Cl, confidence interval; FIGO, International Federation of Gynecology and Obstetrics; HR, hazard ratio.

Table 3 Multivariate Analysis of Relapse-Free and Overall Survival Among Patients with Cervical Cancer $(\mathrm{N}=552)$

\begin{tabular}{|c|c|c|c|c|c|c|}
\hline \multirow[t]{2}{*}{ Variables } & \multicolumn{3}{|c|}{ Relapse-Free Survival } & \multicolumn{3}{|c|}{ Overall Survival } \\
\hline & HR & $95 \% \mathrm{Cl}$ & $P$-value & HR & $95 \% \mathrm{Cl}$ & $P$-value \\
\hline \multicolumn{7}{|l|}{ FIGO stage } \\
\hline||$A \mid$ vs $|B|$ & 3.3 & $1.9-5.6$ & $<0.001$ & 2.6 & I.5-4.5 & $<0.001$ \\
\hline \multicolumn{7}{|l|}{ Nodal metastasis } \\
\hline Positive vs negative & 4.1 & $2.4-7.2$ & $<0.001$ & 3.4 & $2.0-5.7$ & $<0.001$ \\
\hline \multicolumn{7}{|l|}{ B7-H3 } \\
\hline Positive vs negative & 2.1 & $1.2-3.6$ & 0.006 & 1.8 & $1.1-3.0$ & 0.023 \\
\hline
\end{tabular}

Abbreviations: B7-H3, B7 homolog 3; Cl, confidence interval; FIGO, International Federation of Gynecology and Obstetrics; HR, hazard ratio.

patients with non-small cell lung cancer. ${ }^{30}$ Parra et al demonstrated that PD-L1 expression was positively correlated with that of $\mathrm{B} 7-\mathrm{H} 3$ in the TCs of 142 lung adenocarcinomas. ${ }^{31}$ Koyama et al also found that B7H3 positivity was significantly correlated with PD-L1 positivity in patients with upper tract urothelial carcinoma, and their combined expression was significantly associated with poor survival outcomes. ${ }^{32}$ These data indicate that B7-H3 and PD-L1 play nonredundant and somewhat complementary roles in tumor immune evasion, which support the rationale for developing combined therapies that target both proteins. In the study by Yonesaka et al, the dual blockade of B7-H3 and PD-L1 in a murine cancer model yielded a synergistic antitumor effect compared with that when inhibiting either alone. ${ }^{33}$ Enoblituzumab (MGA271), an Fc-optimized monoclonal antibody that selectively targets B7-H3, is currently being evaluated either as a monotherapy or in combination with the PD-1 inhibitor pembrolizumab in Phase 1 studies of patients with B7-H3-expressing cancers (NCT02475213, NCT01391143). Our results support targeting B7-H3 alone or in combination with PD-1/PD-L1 blockade as an immunotherapeutic strategy for the treatment of B7-H3-expressing cervical cancer. Further studies are warranted to identify this protein's cognate receptor(s), the modulation of its expression, and its 
immunomodulatory roles; this will assist in the clinical development of effective immunotherapeutic B7-H3 modulators to treat cancer.

The strengths of our study were that we restricted our analysis to stage IB1/IIA1 squamous cell carcinoma and usual type endocervical adenocarcinoma and that our sample size was relatively large $(\mathrm{N}=552)$. However, our study also had limitations. First, it was retrospective in nature and was therefore subject to inherent unavoidable biases. Second, given intra-tumoral heterogeneity, assessment of immunohistochemical staining using TMAs may not have accurately represented the entire tumor considering marker expression. Finally, the criteria for B7-H3 positivity have not been defined, and the cut-off point we used to stratify positive versus negative expression was based on a previous publication describing a prostate cancer study. ${ }^{6}$ Additional studies from independent cervical cancer cohorts will be required to determine the optimal expression cut-offs for B7-H3 in this particular disease.

\section{Conclusion}

B7-H3 was expressed in the TCs and stromal cells of cervical cancer tissues. The expression of this protein in TCs was more frequent in squamous cell carcinoma, samples that were positive for PD-L1, and samples from patients with lymph node metastasis; moreover, its expression was an independent predictor of poor survival. The frequent expression of B7-H3, PD-L1, or both together supports the development of anti-B7-H3 therapies alone or in combination with PD-1/PD-L1 blockade to treat patients with cervical cancer. Further studies are warranted to validate our results and explore other immunotherapeutic strategies against cervical cancer that include B7-H3 inhibition.

\section{Funding}

This work was supported by grants from the National Key R\&D Program of China (2016YFC1302900 and 2016YFC0902901), National Natural Science Foundation of China (NSFC, 81572559), Key Research Project of Shandong Province (2017CXGC1210), Science and Technology Development Plan of Tai'an (2016NS1099), and Medical and Health Technology Development Plan of Shandong Province (202005010853).

\section{Disclosure}

The authors declare that there are no conflicts of interest regarding the publication of this paper.

\section{References}

1. Siegel RL, Miller KD, Jemal A. Cancer statistics, 2015. CA Cancer J Clin. 2015;65(1):5-29. doi:10.3322/caac.21254

2. Gadducci A, Guerrieri ME, Cosio S. Adenocarcinoma of the uterine cervix: pathologic features, treatment options, clinical outcome and prognostic variables. Crit Rev Oncol Hematol. 2019;135:103-114. doi:10.1016/j.critrevonc.2019.01.006

3. Chung HC, Ros W, Delord JP, et al. Efficacy and safety of pembrolizumab in previously treated advanced cervical cancer: results from the Phase II KEYNOTE-158 Study. J Clin Oncol. 2019;37 (17):1470-1478. doi:10.1200/JCO.18.01265

4. Wang L, Kang FB, Shan BE. B7-H3-mediated tumor immunology: friend or foe? Int J Cancer. 2014;134(12):2764-2771. doi:10.1002/ ijc. 28474

5. Chapoval AI, Ni J, Lau JS, et al. B7-H3: a costimulatory molecule for T cell activation and IFN-gamma production. Nat Immunol. 2001;2 (3):269-274. doi:10.1038/85339

6. Suh WK, Gajewska BU, Okada H, et al. The B7 family member B7-H3 preferentially down-regulates $\mathrm{T}$ helper type 1-mediated immune responses. Nat Immunol. 2003;4(9):899-906. doi:10.1038/ ni967

7. Yim J, Koh J, Kim S, et al. Effects of B7-H3 expression on tumour-infiltrating immune cells and clinicopathological characteristics in non-small-cell lung cancer. Eur $J$ Cancer. 2020;133:74-85. doi:10.1016/j.ejca.2020.03.033

8. Lu Z, Zhao ZX, Cheng P, et al. B7-H3 immune checkpoint expression is a poor prognostic factor in colorectal carcinoma. Mod Pathol. 2020;33(11):2330-2340. doi:10.1038/s41379-020-0587-z

9. Zang X, Thompson RH, Al-Ahmadie HA, et al. B7-H3 and B7x are highly expressed in human prostate cancer and associated with disease spread and poor outcome. Proc Natl Acad Sci U S A. 2007;104 (49):19458-19463. doi:10.1073/pnas.0709802104

10. Crispen PL, Sheinin Y, Roth TJ, et al. Tumor cell and tumor vasculature expression of $\mathrm{B} 7-\mathrm{H} 3$ predict survival in clear cell renal cell carcinoma. Clin Cancer Res. 2008;14(16):5150-5157. doi:10.1158/ 1078-0432.CCR-08-0536

11. Arigami T, Narita N, Mizuno R, et al. B7-h3 ligand expression by primary breast cancer and associated with regional nodal metastasis. Ann Surg. 2010;252(6):1044-1051. doi:10.1097/ SLA.0b013e3181f1939d

12. Brunner A, Hinterholzer S, Riss P, Heinze G, Brustmann H. Immunoexpression of $\mathrm{B} 7-\mathrm{H} 3$ in endometrial cancer: relation to tumor T-cell infiltration and prognosis. Gynecol Oncol. 2012;124 (1):105-111. doi:10.1016/j.ygyno.2011.09.012

13. Zang X, Sullivan PS, Soslow RA, et al. Tumor associated endothelial expression of B7-H3 predicts survival in ovarian carcinomas. Mod Pathol. 2010;23(8):1104-1112. doi:10.1038/modpathol.2010.95

14. Zong L, Zhang M, Wang W, Wan X, Yang J, Xiang Y. PD-L1, B7-H3 and VISTA are highly expressed in gestational trophoblastic neoplasia. Histopathology. 2019;75(3):421-430. doi:10.1111/ his. 13882

15. Huang C, Zhou L, Chang X, Pang X, Zhang H, Zhang S. B7-H3, B7-H4, Foxp3 and IL-2 expression in cervical cancer: associations with patient outcome and clinical significance. Oncol Rep. 2016;35 (4):2183-2190. doi:10.3892/or.2016.4607

16. Han S, Shi X, Liu L, et al. Roles of B7-H3 in cervical cancer and its prognostic value. $J$ Cancer. 2018;9(15):2612-2624. doi:10.7150/ jca. 24959

17. Zong L, Zhang Q, Zhou Y, et al. Expression and significance of immune checkpoints in clear cell carcinoma of the uterine cervix. J Immunol Res. 2020;2020:1283632. doi:10.1155/2020/1283632

18. Han S, Wang Y, Shi X, et al. Negative roles of B7-H3 and B7-H4 in the microenvironment of cervical cancer. Exp Cell Res. 2018;371 (1):222-230. doi:10.1016/j.yexcr.2018.08.014 
19. Aung PP, Parra ER, Barua S, et al. B7-H3 expression in merkel cell carcinoma-associated endothelial cells correlates with locally aggressive primary tumor features and increased vascular density. Clin Cancer Res. 2019;25(11):3455-3467. doi:10.1158/1078-0432.CCR$18-2355$

20. MacGregor HL, Sayad A, Elia A, et al. High expression of B7-H3 on stromal cells defines tumor and stromal compartments in epithelial ovarian cancer and is associated with limited immune activation. $J$ Immunother Cancer. 2019;7(1):357. doi:10.1186/s40425-0190816-5

21. Yuan H, Wei X, Zhang G, Li C, Zhang X, Hou J. B7-H3 over expression in prostate cancer promotes tumor cell progression. J Urol. 2011;186(3):1093-1099. doi:10.1016/j.juro.2011.04.103

22. Zhang J, Liu L, Han S, et al. B7-H3 is related to tumor progression in ovarian cancer. Oncol Rep. 2017;38(4):2426-2434. doi:10.3892/ or.2017.5858

23. Yu TT, Zhang T, Lu X, Wang RZ. B7-H3 promotes metastasis, proliferation, and epithelial-mesenchymal transition in lung adenocarcinoma. Onco Targets Ther. 2018;11:4693-4700. doi:10.2147/OTT.S169811

24. Wang R, Ma Y, Zhan S, et al. B7-H3 promotes colorectal cancer angiogenesis through activating the $\mathrm{NF}-\kappa \mathrm{B}$ pathway to induce VEGFA expression. Cell Death Dis. 2020;11(1):55. doi:10.1038/ s41419-020-2252-3

25. Brustmann H, Igaz M, Eder C, Brunner A. Epithelial and tumor-associated endothelial expression of B7-H3 in cervical carcinoma: relation with $\mathrm{CD} 8+$ intraepithelial lymphocytes, FIGO stage, and phosphohistone $\mathrm{H} 3$ (PHH3) reactivity. Int J Gynecol Pathol. 2015;34(2):187-195. doi:10.1097/PGP.0000000000000116

26. Yang J, Zong L, Wang J, Wan X, Feng F, Xiang Y. Epithelioid Trophoblastic Tumors: treatments, Outcomes, and Potential Therapeutic Targets. J Cancer. 2019;10(1):11-19. doi:10.7150/ jca. 28134
27. Heeren AM, Punt S, Bleeker MC, et al. Prognostic effect of different PD-L1 expression patterns in squamous cell carcinoma and adenocarcinoma of the cervix. Mod Pathol. 2016;29(7):753-763. doi:10.1038/modpathol.2016.64

28. Kawachi A, Yoshida H, Kitano S, Ino Y, Kato T, Hiraoka N. Tumorassociated CD204(+) M2 macrophages are unfavorable prognostic indicators in uterine cervical adenocarcinoma. Cancer Sci. 2018;109 (3):863-870. doi:10.1111/cas.13476

29. Enwere EK, Kornaga EN, Dean M, et al. Expression of PD-L1 and presence of CD8-positive $\mathrm{T}$ cells in pre-treatment specimens of locally advanced cervical cancer. Mod Pathol. 2017;30(4):577-586. doi:10.1038/modpathol.2016.221

30. Altan M, Pelekanou V, Schalper KA, et al. B7-H3 Expression in NSCLC and Its Association with B7-H4, PD-L1 and Tumor-Infiltrating Lymphocytes. Clin Cancer Res. 2017;23 (17):5202-5209. doi:10.1158/1078-0432.CCR-16-3107

31. Parra ER, Villalobos $\mathrm{P}$, Zhang $\mathrm{J}$, et al. Immunohistochemical and Image Analysis-Based Study shows that several immune checkpoints are co-expressed in non-small cell lung carcinoma tumors. J Thorac Oncol. 2018;13(6):779-791. doi:10.1016/j.jtho.2018.03.002

32. Koyama Y, Morikawa T, Miyama Y, et al. B7-H3 expression in upper tract urothelial carcinoma associates with adverse clinicopathological features and poor survival. Pathol Res Pract. 2020;216(12):153219. doi:10.1016/j.prp.2020.153219

33. Yonesaka K, Haratani K, Takamura S, et al. B7-H3 negatively modulates CTL-mediated cancer immunity. Clin Cancer Res. 2018;24 (11):2653-2664. doi:10.1158/1078-0432.CCR-17-2852
OncoTargets and Therapy

\section{Publish your work in this journal}

OncoTargets and Therapy is an international, peer-reviewed, open access journal focusing on the pathological basis of all cancers, potential targets for therapy and treatment protocols employed to improve the management of cancer patients. The journal also focuses on the impact of management programs and new therapeutic

Submit your manuscript here: https://www.dovepress.com/oncotargets-and-therapy-journ agents and protocols on patient perspectives such as quality of life, adherence and satisfaction. The manuscript management system is completely online and includes a very quick and fair peer-review system, which is all easy to use. Visit http://www.dovepress.com/ testimonials.php to read real quotes from published authors. 